\title{
SUPPLY CHAIN MANAGEMENT IN SMALL AND MEDIUM-SIZED ENTERPRISES
}

\author{
Peter Nyhuis, Katja Hasenfuß \\ Institut of Production Systems and Logistics (IFA), University of Hannover. Email: \\ nyhuis@ifa.uni-hannover.de.
}

\begin{abstract}
In the production logistics context, the success of supply chains corresponds to their ability to meet the logistic performance expectations of customers. This ability depends on the contributions of all supply chain members to the logistical performance of the entire chain. Moreover the overall performance is affected by the dynamic interrelationships between the specific logistic performances of the separate supply chain members. This includes small and medium-sized companies (SMEs). The paper mainly focuses on current supply chain management methods and supply chain management concepts to improve logistical performance and their present application in today's industries especially SMEs.
\end{abstract}

Key words: supply chain management (SCM), logistical performance, SCM-methods, small and medium-sized companies (SMEs)

\section{INTRODUCTION}

Confronted with constant structural changes in society and the manufacturing industry, enterprises have undertaken several innovative activities to optimize their processes and to improve their logistical performance. There is hardly any enterprises still regard and treat logistics as a function of simply transportation and stock-keeping which may not influence their competitiveness. Rather, most enterprises see that their logistics significantly supports their business strategy. In some enterprises their business strategy even arises, at least partly, out of their logistic strengths. In continuation of this trend enterprises cooperate closely with their suppliers as well as with their customers in order to improve business

Please use the following format when citing this chapter:

Nyhuis, Peter, Hasenfuß, Katja, 2006, in International Federation for Information Processing (IFIP), Volume 207, Knowledge Enterprise: Intelligent Strategies In Product Design, Manufacturing, and Management, eds. K. Wang, Kovacs G., Wozny M., Fang M., (Boston: Springer), pp. 386-392. 
performance. This enables them to further focus on core competencies. Originally, this led to the forming of supply chains, which are characterized by the share of information between suppliers and producers. An intensive flow of data helps the enterprises of a supply chain to plan more accurately and to adjust their capacities more effectively, since information is provided both earlier and in more detail. Thus, supply chains facilitate the avoidance of large deviations of demand along the value added chain as described by Forrester (1961).

\section{SUPPLY CHAIN MANAGEMENT}

The understanding of SCM is ever-changing. Still SCM is intensively and controversial discussed in respect of its content regarding practical experiences in a fast developing business environment. Nevertheless, current definitions of SCM emphasize the collaborative aspects, that this management paradigm incorporates - on a strategic (Schönsleben, 2000) as well as on an operational level (Wiendahl et al., 1998). Hieber and Alard (2000) state that there is still a gap between the conceptual ideas of supply chain management and its application in industry. This is especially true for the case of SMEs. There are still disparities between the best practices of SCM and their implementation in practice: enterprises in supply chains pursue individual rather than common objectives. Nevertheless SCM promises more effective processes, better customer orientation and lower costs.

\subsection{Concepts and Methods}

SCM is internationally accepted by research and industrial practice as one element of the business strategy. Within the scope of SCM it is essential to operationalize the objectives which have been defined within the supply chain strategy. For this purpose relevant functions and tasks can be differentiated by their planning horizons and their objective targets. Therefore, a number of different SCM-concepts and SCM-methods as well as Best Practice examples exist to realize the possible potentials in supply chains (Hieber 2002). As a part of a research project on network-oriented production management accomplished at the Institute of Production Systems and Logistics (IFA), a number of theoretical methods from the fields of production planning and control, such as the funnel model, throughput diagrams and logistic operating curves, have been adopted for production management in a network of several partners (Wiendahl et. al., 1998). Based on the supply chain planning matrix (Meyr 2002) and the SCM-function 
model of the RWTH Aachen University a categorization scheme was developed by the IFA as a part of a current research project on methods for cooperative business processes. The new categorisation scheme is shown in figure 1. It includes the functions SC-Configuration, SC-Planning, SCExecution and SC-Controlling. In this scheme the four SCM-functions are arranged accordingly to their planning horizon.

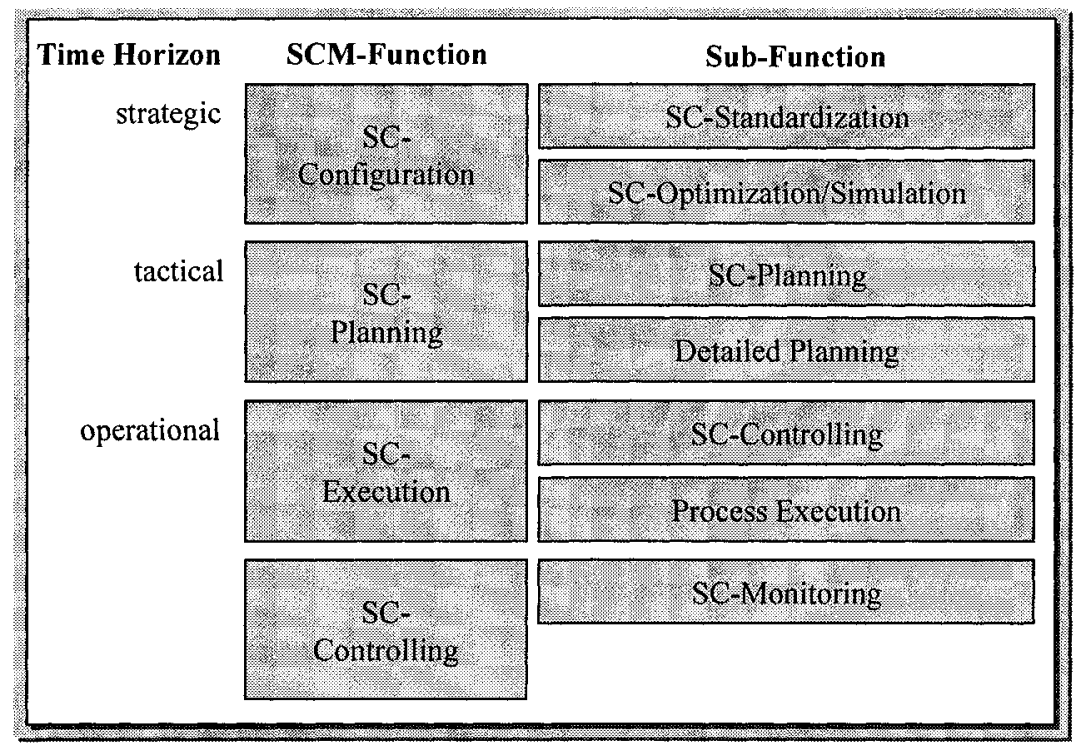

Figure 1. SCM categorization scheme

\subsection{Application of Supply Chain Management}

To analyze the actual status of application and implementation of SCM best practices in industry a survey in German speaking countries was conducted by the ETH-Zurich and the IFA in autumn 2005. Some 51 questionnaires have been analyzed on the subject ,supply chain management and business success" (Schnetzler et al. 2006). Enterprises from different branches and of different sizes took part in the survey. About $30 \%$ of the enterprises are SMEs. The European SME definition set the upper limit of employees to 250 and the annual turnover at a maximum of $€ 50$ million or the annual balance sheet total at a maximum of $€ 43$ million. $20 \%$ of all enterprises of the conducted survey have 250 to 500 employees. Figure 2 shows a fairly heterogeneous distribution of different best practices. The analysis of the survey showed that only $30 \%$ of the enterprises apply for example order promising, meaning available/capable to promise and that it is 
also seldom planned to implement it. This is astonishing especially as current SCM-/ERP-Software provides this functions. With this best practice enterprises would be able to offer a more realistic order confirmation. This would lead to an increase of their delivery reliability and consequently, to an improvement of their logistical performance. It is especially relevant if enterprises produce by the principle of make to order - which most of the questioned enterprises do.

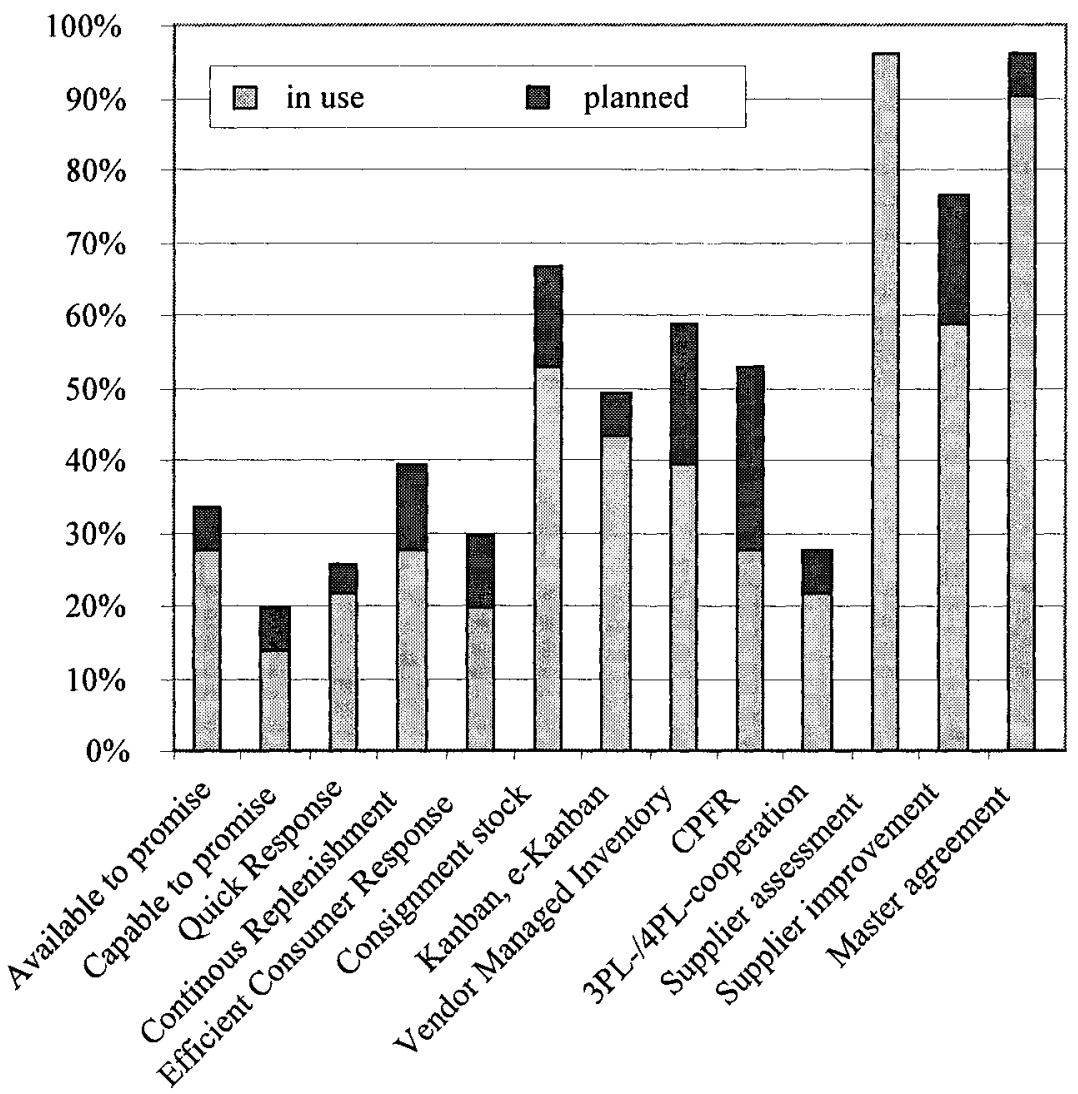

Figure 2. Extract of best practices in use and planned

Furthermore, the following supply concepts where analyzed in the survey: quick response $(\mathrm{QR})$, continuous replenishment (CR), efficient consumer response (ECR), consignment stock, kanban und vendor managed inventory (VMI). The three first supply concepts are most commonly in use. This may be due to the fact that $\mathrm{QR}, \mathrm{CR}$ and ECR were originally developed for trading. In many cases enterprises plan to implement monitoring, controlling 
or benchmarking concepts to record, evaluate and compare the performance of their SCM. The analysis of the survey showed, that the implementation of best practices in the German-speaking countries is still not widely spread. The main obstacle seams to be the scarcity of resources. This comes along with the incompatibility of the present information systems. Furthermore SMEs denominate the name recognition of SCM-methods and SCMconcepts as rather low. Therefore, the selection of suitable concepts and methods obviously becomes extremely challenging for SMEs. The planning and implementation of suitable SCM-concepts and SCM-methods becomes even more difficult due to the fact that they do not have the required knowhow and responsibilities for implementation are not defined.

\section{SUPPLY CHAIN MANAGEMENT IN SMES}

A differentiated analysis of the goals of SMEs shows that cooperating SMEs seldom possess one consistent strategy. In addition if individual SMEs do have a SCM-strategy, they often do not derivate their individual objectives from this strategy. Their concentration on core competences interferes with strategic and policy. It also explains the multiplicity of varying SCM-objectives. Most of those objectives focus on operational functions. Those operational objectives may be structured by the SCOR processes source, make and deliver. Out of all objectives the logistical objectives can be consolidated to the logistical objectives of supply chains. These are short delivery time, high service level, even machine utilization and low work in progress and stock. Therefore they are looking for simple but effective and efficient methods or concepts to conduct the vitally important SCM-functions, which shall improve their logistic performance.

\subsection{Restrictions and Requirements of SMEs}

Since the application of SCM-concepts and SCM-methods is not that widespread, it was investigated, which restrictions and requirements of SMEs are existent. The determined restrictions in the requirement analysis may be split up into the categories transparency, cooperation, realization and costs. In the category transparency it was for example discovered that beside the fact that different SCM-concepts and SCM-methods are not well known like mentioned before companies do quite often neither know the field of application of a known SCM-concept or SCM-method nor do they know which benefit they may generate out of the employment of a specific concept or method. One reason among others might be that different 
management approaches are subsumed in SCM-concepts. Therefore it is difficult to get a general conspectus on existing concepts and methods.

\section{CONCLUSION}

Concluding one may state that from a logistical point of view the advantages of SCM are obvious and demonstrated by the results of SCM in industrial practice. However there is still a need for further implementation into practice and further development which will be executed in research and in industrial practice. The demand for SME oriented SCM-concepts and SCM-methods may be explained by the existent inherent difference between major enterprises and SMEs. Furthermore, the characteristic comprehension and definition of SCM differs as well as the particular goals of these enterprises. Usually, SMEs state that they need to focus on their core competencies. Consequently, many SMEs expect to get operational assistance in their day to day business. Both realization and application of SCM-concepts and SCM-methods demands the acceptance of the employees, therefore and because of the lack of resources SMEs prefer simple methods and concepts which require less specialized knowledge. Thus a guideline to support cooperative business processes of SMEs shall be developed within the current research project at the IFA. This guideline will help SMEs to select suitable SCM-concepts and SCM-methods and to pre-estimate the benefit of the selected concept or method.

\section{REFERENCES}

1. Forrester, J. W., (1961), Industrial Dynamics, John Wiley \& Sons, New York.

2. Hieber, R., (2002): Supply chain management. Zürich.

3. Hieber, R., Alard, R., (2000), Supply Chain Management - A Survey of Practices and Trends in Swiss Industry. In: Strandhagen, J.O.; Alfnes, E. (Eds.): Information and Communication technology (ICT) in Logistics and Production Management. Proceedings of the Annual IFIP WG 5.7 Working Conference, Conference Proceedings 2000. Tromsö, Norway, 28-30 June 2000.

4. Meyr, H., Wagner, M., Rohde, J., (2002), Structure of Advanced Planning Systems. In: Stadtler, H., Kilger, C., Supply Chain Management and Advanced Planning - Concepts, Models, Software and Case Studies. Berlin, Heidelberg, New York. 
5. Schönsleben, P., 2000, Integral Logistics Management - Planning and Control of Comprehensive Business Processes. St. Lucie Press, Boca Raton et al.

6. Wiendahl, H.-P., Hoebig, M., Kuhn, A., et al., 1998, Kennzahlengestützte Prozesse im Supply Chain Management. In: Industrie Management, Vol. 14, No. 6, pp. 18-24.

7. Schnetzler, M., Nölle, A., Hasenfuss, K., et al., 2006, SCM und Unternehmenserfolg. Zürich. 